\title{
Interactive comment on "The impact of fluctuations and correlations in droplet growth by collision-coalescence revisited. Part I: Numerical calculation of post-gel droplet size distribution" by Lester Alfonso and Graciela B. Raga
}

\section{Anonymous Referee \#1}

Received and published: 29 December 2016

This paper applies the theory of finite discrete aggregation to predict condensation in small systems of aggregating droplets. This is a very interesting application of a stochastic process to small systems under the premise that the finite size of the system leads to behavior that is quite distinct from that of an infinite system under the same physical processes, particularly with respect to the emergence of a gel phase. I am not qualified to comment on the science of cloud physics but with respect to stochastic aggregation I have a few comments:

1. A system with five initial particles is indeed very small (Fig 2). I assume this result is 
shown for the purpose of demonstrating the method, it is confusing, however, because the system is too small to be of relevance.

2. The Tanaka/Nakazawa equation, which the authors use to calculate the gel mass, is not approximate. It requires, however, a trial-and-error procedure to determine the correct value of i_1 in Eqs 14 and 15. This is because Tanaka/Nakazawa depends on the mean distribution to obtain the gel fraction. If one has access to individual distributions, as the authors have, there is a simpler method: calculate the maximum mass in each individual distribution and obtain their average by weighting each value by the probability of the distribution.

3. Finite size is not necessary for gelation to occur. The solutions converge when the size of the system becomes large, though these solutions cannot be obtained by traditional KCE and instead require the methods of Lushnikov (2004) or Matsoukas (2015). This means that finite systems, even with as few particles as 40 , may be adequately described by these solutions without the need for a detailed (and costly) solution of the master equation.

4. The complete phase space of a population with $\mathrm{N}=40$ monomers contains 37338 states. Do the authors solve the Master equation for all of these? Some details need to be offered regarding this calculation.

This work is a novel application of stochastic dynamics in finite systems. It highlights the fact that reliance on the average behavior of a complex system, while sufficient for a large number of physical systems, is inadequate in populations that undergo a phase transition.

Interactive comment on Atmos. Chem. Phys. Discuss., doi:10.5194/acp-2016-922, 2016. 